\title{
Recursive Least-Squares Fixed-Interval Smoother Using Covariance Information based on Innovation Approach in Linear Continuous Stochastic Systems
}

\author{
Seiichi Nakamori \\ Professor Emeritus, Kagoshima University, Kourimoto, Kagoshima, Japan \\ Email: k6165161@kadai.jp
}

\begin{abstract}
This paper newly proposes the recursive least-squares (RLS) fixed-interval smoother and filter, based on the innovation theory, in linear continuous-time stochastic systems. It is assumed that the signal is observed with additive white noise and the signal process is uncorrelated with the observation noise. It is a characteristic that the estimators use the covariance function of the signal, in the form of the semi-degenerate kernel, and the variance of the observation noise. Also, the algorithm for the estimation error variance function of the RLS fixed-interval smoother is developed to validate the stability of the proposed fixed-interval smoother. The numerical simulation example shows that the estimation accuracy of the proposed fixed-interval smoother is superior to that of the existing fixed-interval smoother using the covariance information.
\end{abstract}

Keywords: RLS Wiener estimators, fixed-interval smoother, innovation approach, covariance information, linear continuous-time systems.

\section{Introduction}

Hitherto, there have been a lot of researches on the estimation problems using the state-space model [1], [2]. Recursive least-squares (RLS) Wiener fixed-point smoother [3] is also studied in linear continuoustime stochastic systems. It is interesting that the RLS Wiener estimators use the information of the auto-variance function of the state vector besides the observation matrix and the system matrix in the state-space model.

In [4], [5], recursive fixed-lag smoother, using the covariance information, is studied in linear discretetime systems. It is a characteristic that the auto-covariance function of the signal is expressed in the semi-degenerate kernel form. In [6], the RLS Wiener fixed-lag smoother is developed in linear discrete-time stochastic systems. In [7], assuming that the signal process is modelled in terms of the autoregressive model, the RLS Wiener fixed-lag smoother is proposed in linear discrete-time stochastic systems.

In [8], in terms of the state-space models, the smoothing algorithms are developed based on the innovation theory. In [9], the optimal fixed-lag smoother and its error variance matrix are derived for the continuous linear systems with the white measurement noise. In [10], the RLS fixed-interval smoother, using the covariance information, is designed based on the innovation approach in linear continuous-time stochastic systems. Here, it is assumed that the observation noise is white. In [11], with the state-space model, the RLS fixed-interval smoother is proposed based on the innovation approach. In [12], based on the innovation theory, the RLS fixed-interval smoother, using the covariance information, is proposed in linear discrete-time stochastic systems. Also, in [13], based on the innovation theory, the RLS Wiener fixed-interval smoothing algorithm is presented for the measurement with colored observation noise.

This paper, by correcting the expression of the impulse response function in [10], newly proposes the RLS fixed-interval smoother and filter, based on the innovation theory, in linear continuous-time stochastic systems. It is assumed that the signal is observed with additive white noise and the signal process is uncorrelated with the observation noise. It is a characteristic that the estimators use the covariance function of the signal, in the form of the semi-degenerate kernel, and the variance of the observation noise. The fixed-interval smoothing and filtering algorithms are presented in Theorem 1 . The corrected point in the expression of the impulse response function from that in the fixed-interval in [10] is clarified. The details are discussed after the proof of Theorem 1. The fixed-interval smoothing algorithm, proposed 
in this paper, is regarded as the continuous-time version of the fixed-interval smoother [12] in linear discrete-time stochastic systems. Also, the algorithm for the estimation error variance function of the RLS fixed-interval smoother is developed to validate the stability of the proposed fixed-interval smoother.

In the numerical example of section 5, the estimation accuracy of the proposed fixed-interval smoother is compared with that in [10].

\section{Fixed-interval smoothing problem}

Let an observation equation be given by

$$
y(t)=z(t)+v(t)
$$

in linear continuous-time stochastic systems, where $z(t)$ is an $\mathrm{m} \times 1$ signal vector and $v(t)$ is white observation noise. It is assumed that the signal and the observation noise are mutually independent stochastic processes with zero means. Let the auto-covariance function of $v(t)$ be given by

$$
E\left[v(t) v^{T}(s)\right]=R \delta(t-s), R>0 .
$$

Here, $\delta(\cdot)$ denotes the Dirac $\delta$ function.

Let $K(t, s)$ represent the auto-covariance function of the signal process and let $K(t, s)$ be expressed in the semi-degenerate kernel form [9], [10] of

$$
K(t, s)=\left\{\begin{array}{l}
A(t) B^{T}(s), 0 \leq s \leq t \\
B(t) A^{T}(s), 0 \leq t \leq s .
\end{array}\right.
$$

Here, $A(t)$ and $B(s)$ are bounded $\mathrm{m} \times p$ matrices.

Let a fixed-interval smoothing estimate $\hat{z}(t \mid T)$ of $z(t)$ be given by

$$
\hat{z}(t \mid T)=\int_{0}^{T} g(t, s) v(s) \mathrm{d} s
$$

as a linear integral transformation of the innovation process $v(\mathrm{~s})=\mathrm{y}(\mathrm{s})-\hat{z}(s \mid s), 0 \leq s \leq T$, where $\mathrm{g}(t, s), \hat{z}(s \mid s)$ and $\mathrm{T}$ are referred to as the impulse response function, the filtering estimate of $\mathrm{z}(\mathrm{s})$ and the fixed interval respectively.

The impulse response function, which minimizes the mean-square value of the fixed-interval smoothing error $\mathrm{z}(t)-\hat{z}(t \mid T)$,

$$
J=E\left[\|z(t)-\hat{z}(t \mid T)\|^{2}\right]
$$

satisfies

$$
\begin{aligned}
& E\left[z(t) v^{T}(s)\right]=\int_{0}^{T} g(t, \tau) E\left[v(\tau) v^{T}(s)\right] \mathrm{d} \tau \\
& =g(t, s) R
\end{aligned}
$$

by an orthogonal projection lemma [14]

$$
z(t)-\hat{z}(t \mid T) \perp v(s), 0 \leq s, t \leq T .
$$

Here, " $\perp$ " denotes the notation of the orthogonality. From (1), (2) and (6), the impulse response function, for the linear least-squares fixed-interval smoothing estimates, satisfies

$$
\begin{aligned}
& g(t, s) R=E\left[z(t)(y(s)-\hat{z}(s \mid s))^{T}\right] \\
& =K(t, s)-\int_{0}^{s} E\left[z(t) v^{T}(\tau)\right] g^{T}(s, \tau) \mathrm{d} \tau \\
& =K(t, s)-\int_{0}^{s} g(t, \tau) R g^{T}(s, \tau) \mathrm{d} \tau .
\end{aligned}
$$

Based on the preliminary formulation on the fixed-interval smoothing problem, the fixed-interval smoothing and filtering algorithms are proposed in Theorem 1. 


\section{$3 \quad$ Fixed-interval smoothing and filtering algorithms}

The filtering and fixed-interval smoothing algorithms based on the innovation theory is presented in Theorem 1. The algorithms are derived with the invariant imbedding method [10] in linear continuous stochastic system.

Theorem 1 Let the observation equation be given by (1). Let the auto-covariance function of the signal $z(t)$ be given by (3) in the semi-degenerate kernel form in linear continuous-time stochastic systems. Then the RLS algorithms for the fixed-interval smoothing estimate $\hat{z}(t \mid T)$ and the filtering estimate $\hat{z}(t \mid t)$ of $z(t)$ consist of $(9)-(15)$.

Fixed-interval smoothing estimate $\hat{z}(t \mid T)$ of $\mathrm{z}(\mathrm{t})$ :

$$
\begin{gathered}
\hat{z}(t \mid T)=\hat{z}(t \mid t)+\left(B(t)-A(t) \mathrm{q}_{0}(\mathrm{t})\right) e(t \mid T) \\
\frac{d e(t \mid T)}{d t}=-\mathrm{J}(\mathrm{t}, \mathrm{t})(\mathrm{y}(\mathrm{t})-\hat{z}(t \mid t))+J(t, t) R \mathrm{~J}_{0}^{\mathrm{T}}(\mathrm{t}) e(t \mid T), \quad \mathrm{e}(\mathrm{T} \mid \mathrm{T})=0 \\
J(t, t)=A^{T}(t) R^{-1}
\end{gathered}
$$

Filtering estimate $\hat{z}(t \mid t)$ of $z(t)$ :

$$
\begin{gathered}
\hat{z}(t \mid t)=A(t) e_{0}(t) \\
\frac{d \mathrm{e}_{0}(t)}{d t}=\mathrm{J}_{0}(\mathrm{t}) \nu(\mathrm{t})=\mathrm{J}_{0}(\mathrm{t})(\mathrm{y}(\mathrm{t})-\hat{z}(t \mid t)), \mathrm{e}_{0}(0)=0 \\
\frac{d \mathrm{q}_{0}(t)}{d t}=\mathrm{J}_{0}(t) R \mathrm{~J}_{0}^{\mathrm{T}}(t), \mathrm{q}_{0}(0)=0 \\
\mathrm{~J}_{0}(t)=\left(B^{T}(t)-\mathrm{q}_{0}(t) A^{T}(t)\right) R^{-1}
\end{gathered}
$$

Proof. The expression for the fixed-interval smoothing estimate in (4) is written as

$$
\hat{z}(t \mid T)=\int_{0}^{t} g(t, s) v(s) \mathrm{d} s+\int_{t}^{T} g(t, s) v(s) \mathrm{d} s .
$$

The first term on the right hand side of (16) represents the filtering estimate $\hat{z}(t \mid t)$ of $\mathrm{z}(t)$ and the second term the correction quantity of the fixed-interval smoothing estimate to the filtering estimate.

At first, for $0 \leq s \leq t$, from (3) and (8), the impulse response function $\mathrm{g}(t, s)$ for the filtering estimate $\hat{z}(t \mid t)$ satisfies

$$
g(t, s) R=A(t) B^{T}(s)-\int_{0}^{s} g(t, \tau) R(\tau) g^{T}(s, \tau) \mathrm{d} \tau .
$$

Introducing an auxiliary function $J_{0}(s)$, which satisfies

$$
J_{0}(s) R=B^{T}(s)-\int_{0}^{s} J_{0}(\tau) R(\tau) g^{T}(s, \tau) \mathrm{d} \tau,
$$

we get

$$
g(t, s)=A(t) J_{0}(s), \quad 0 \leq s \leq t \leq T .
$$

Substituting (19) into (18) and introducing a function 


$$
q_{0}(s)=\int_{0}^{s} J_{0}(\tau) R J_{0}^{T}(\tau) \mathrm{d} \tau
$$

we have

$$
\mathrm{J}_{0}(s) R=B^{T}(s)-\int_{0}^{s} \mathrm{~J}_{0}(\tau) R(\tau) J_{0}^{T}(\tau) A^{T}(s) \mathrm{d} \tau=B^{T}(s)-\mathrm{q}_{0}(s) A^{T}(s) .
$$

Differentiating (20) with respect to s, we obtain

$$
\frac{d \mathrm{q}_{0}(s)}{\mathrm{d} s}=\mathrm{J}_{0}(s) R \mathrm{~J}_{0}^{\mathrm{T}}(s), \mathrm{q}_{0}(0)=0 .
$$

Let us introduce a function

$$
\mathrm{e}_{0}(\mathrm{t})=\int_{0}^{t} \mathrm{~J}_{0}(\tau) \nu(\tau) \mathrm{d} \tau
$$

From (16) and (19), the filtering estimate $\hat{z}(t, t)$ is given by

$$
\hat{z}(t, t)=\int_{0}^{t} g(t, \tau) \nu(\tau) \mathrm{d} \tau=\int_{0}^{t} A(t) \mathrm{J}_{0}(\tau) \nu(\tau) \mathrm{d} \tau=A(t) e_{0}(t) .
$$

Differentiating (23) with respect to t, we have

$$
\frac{d \mathrm{e}_{0}(t)}{d t}=\mathrm{J}_{0}(\mathrm{t}) \nu(\mathrm{t})=\mathrm{J}_{0}(\mathrm{t})(\mathrm{y}(\mathrm{t})-\hat{z}(t, t)), \mathrm{e}_{0}(0)=0 .
$$

Secondly, for $0 \leq \mathrm{t} \leq \mathrm{s} \leq \mathrm{T}$, from (3), (8), (19) and (20), $\mathrm{g}(\mathrm{t}, \mathrm{s})$ satisfies

$$
\begin{aligned}
& g(t, s) R=B(t) A^{T}(s)-\int_{0}^{t} g(t, \tau) R g^{T}(s, \tau) \mathrm{d} \tau \\
& -\int_{t}^{s} g(t, \tau) R g^{T}(s, \tau) \mathrm{d} \tau \\
& =B(t) A^{T}(s)-\int_{0}^{t} A(t) J_{0}(\tau) R J_{0}^{T}(\tau) A^{T}(\tau) \mathrm{d} \tau \\
& -\int_{t}^{s} g(t, \tau) R J_{0}^{T}(\tau) A^{T}(\tau) \mathrm{d} \tau \\
& =\left(B(t)-A(t) q_{0}(t)\right) A^{T}(s)-\int_{t}^{s} g(t, \tau) R J_{0}^{T}(\tau) A^{T}(\tau) \mathrm{d} \tau .
\end{aligned}
$$

Introducing an auxiliary function $J(t, s)$, which satisfies

$$
J(t, s) R=A^{T}(s)-\int_{t}^{s} J(t, \tau) R \mathrm{~J}_{0}^{\mathrm{T}}(\tau) \mathrm{d} \tau A^{T}(s)
$$

we obtain

$$
g(t, s)=\left(B(t)-A(t) \mathrm{q}_{0}(\mathrm{t})\right) \mathrm{J}(\mathrm{t}, \mathrm{s}), \quad 0 \leq t \leq s \leq T .
$$

From (16) and (28), we have an expression for the fixed-interval smoothing estimate as follows.

$$
\begin{aligned}
& \hat{z}(t \mid T)=\hat{z}(t \mid t)+\int_{t}^{T} g(t, s) v(s) \mathrm{d} s \\
& =\hat{z}(t \mid t)+\left(B(t)-A(t) q_{0}(t)\right) \int_{t}^{T} J(t, s) v(s) \mathrm{d} s \\
& =\hat{z}(t \mid t)+\left(B(t)-A(t) q_{0}(t)\right) e(t \mid T)
\end{aligned}
$$

Here, we introduced the function $e(t \mid T)$ expressed by

$$
e(t \mid T)=\int_{t}^{T} \mathrm{~J}(\mathrm{t}, \mathrm{s}) v(s) \mathrm{d} s
$$

Differentiating (27) with respect to t, we have 


$$
\frac{\partial J(t, s)}{\partial t} R=J(t, t) R \mathrm{~J}_{0}^{\mathrm{T}}(\mathrm{t}) A^{T}(s)-\int_{t}^{s} \frac{\partial J(t, \tau)}{\partial t} R \mathrm{~J}_{0}^{\mathrm{T}}(\tau) \mathrm{d} \tau A^{T}(s) .
$$

From (27) and (31), we obtain

$$
\frac{\partial J(t, s)}{\partial t}=J(t, t) R \mathrm{~J}_{0}^{\mathrm{T}}(\mathrm{t}) J(t, s) .
$$

Differentiating (30) with respect to t and using (32), we get

$$
\begin{aligned}
& \frac{d e(t \mid T)}{d t}=-J(t, t) v(t)+\int_{t}^{T} \frac{\partial J(t, s)}{\partial t} v(s) \mathrm{d} s \\
& =-J(t, t) v(t)+J(t, t) R \mathrm{~J}_{0}^{\mathrm{T}}(t) \int_{t}^{T} J(t, s) v(s) \mathrm{d} s \\
& =-J(t, t)(y(t)-\hat{z}(t \mid t))+J(t, t) R \mathrm{~J}_{0}^{\mathrm{T}}(t) e(t \mid T), e(T \mid T)=0 .
\end{aligned}
$$

From $(27), \mathrm{J}(\mathrm{t}, \mathrm{t})$ is given by $J(t, t)=A^{T}(t) R^{-1}$.

In (9), the fixed-interval smoothing estimate $\hat{z}(t \mid T)$ is given as a sum of the filtering estimate $\hat{z}(t \mid t)$ and the quantity $\left(B(t)-A(t) \mathrm{q}_{0}(\mathrm{t})\right) e(t \mid T) . e(t \mid T)$ is calculated recursively by (10) in the reverse direction of time $\mathrm{t}$, starting with the condition $\mathrm{e}(\mathrm{T} \mid \mathrm{T})=0$ at $\mathrm{t}=\mathrm{T}$ of $\mathrm{e}(\mathrm{t} \mid \mathrm{T})$. The filtering estimate is calculated recursively by (12) with (13)-(15), starting with the initial conditions $\mathrm{e}_{0}(0)=0$ and $\mathrm{q}_{0}(0)=0$.

As shown in (19) and (28), in the proof of Theorem 1, the impulse response function $\mathrm{g}(\mathrm{t}, \mathrm{s})$ has different expressions for $0 \leq \mathrm{s} \leq \mathrm{t} \leq \mathrm{T}$ and $0 \leq \mathrm{t} \leq \mathrm{s} \leq \mathrm{T}$. However, in [10], $\mathrm{g}(\mathrm{t}, \mathrm{s})$ has the same expression for both $0 \leq \mathrm{s} \leq \mathrm{t} \leq \mathrm{T}$ and $0 \leq \mathrm{t} \leq \mathrm{s} \leq \mathrm{T}$. In this paper, this erroneous expression for $\mathrm{g}(\mathrm{t}, \mathrm{s})$ in [10] is corrected.

Section 4 proposes the fixed-interval smoothing error variance function of the fixed-interval smoother proposed in Theorem 1.

\section{Fixed-interval smoothing error variance function}

Concerning $\mathrm{g}(\mathrm{t}, \mathrm{s})$ of the first term in $(16), \mathrm{g}(\mathrm{t}, \mathrm{s}) \mathrm{R}$ represents the filtering error covariance function $\mathrm{E}\left[(\mathrm{z}(\mathrm{t})-\hat{z}(t \mid t))(z(s)-\hat{z}(s \mid s))^{T}\right]$. Similarly, regarding $\mathrm{g}(\mathrm{t}, \mathrm{s}), 0 \leq \mathrm{t}, \mathrm{s} \leq \mathrm{T}$, in $(8), \mathrm{g}(\mathrm{t}, \mathrm{s}) \mathrm{R}$ represents the smoothing error covariance function $\mathrm{E}\left[(\mathrm{z}(\mathrm{t})-\hat{z}(t \mid T))(z(s)-\hat{z}(s \mid T))^{T}\right]$.

Referring to Theorem 1, the fixed-interval smoothing error variance function $\mathrm{P}(\mathrm{t} \mid \mathrm{T})$ is formulated as

$$
\begin{aligned}
& \mathrm{P}(\mathrm{t} \mid \mathrm{T})=\mathrm{E}\left[(\mathrm{z}(\mathrm{t})-\hat{z}(\mathrm{t} \mid \mathrm{T}))(\mathrm{z}(\mathrm{t})-\hat{z}(\mathrm{t} \mid \mathrm{T}))^{T}\right] \\
& =\mathrm{K}(\mathrm{t}, \mathrm{t})-\mathrm{E}\left[\hat{z}(\mathrm{t} \mid \mathrm{T}) \hat{z}^{T}(\mathrm{t} \mid \mathrm{T})\right] \\
& =\mathrm{K}(\mathrm{t}, \mathrm{t})-\mathrm{E}\left[\left(\hat{z}(\mathrm{t} \mid \mathrm{t})+\left(B(t)-A(t) \mathrm{q}_{0}(\mathrm{t})\right) e(t \mid T)\right)\left(\left(\hat{z}(\mathrm{t} \mid \mathrm{t})+\left(B(t)-A(t) \mathrm{q}_{0}(\mathrm{t})\right) e(t \mid T)\right)^{T}\right]\right. \\
& =\mathrm{K}(\mathrm{t}, \mathrm{t})-\mathrm{E}\left[\hat{z}(\mathrm{t} \mid \mathrm{t}) \hat{z}^{T}(\mathrm{t} \mid \mathrm{t})\right]-\left(B(t)-A(t) \mathrm{q}_{0}(\mathrm{t})\right) E\left[e(t \mid T) e^{T}(t \mid T)\right]\left(B(t)-A(t) \mathrm{q}_{0}(\mathrm{t})\right)^{T} .
\end{aligned}
$$

Here, from (30) and the independence property of the filtering estimate with the innovation process, we see that $\hat{z}(\mathrm{t} \mid \mathrm{t})$ is independent from $e(t \mid T)$ as

$$
\begin{aligned}
& \mathrm{E}\left[\hat{z}(\mathrm{t} \mid \mathrm{t}) e^{T}(\mathrm{t} \mid \mathrm{T})\right]=\mathrm{E}\left[\hat{z}(\mathrm{t} \mid \mathrm{t})\left(\int_{t}^{T} \mathrm{~J}(\mathrm{t}, \mathrm{s}) v(s) \mathrm{d} s\right)^{T}\right] \\
& =\int_{t}^{T} \mathrm{E}\left[\hat{z}(\mathrm{t} \mid \mathrm{t}) v^{T}(s)\right] J^{T}(t, s) \mathrm{d} s \\
& =0 .
\end{aligned}
$$

Also, from (30), $E\left[e(t \mid T) e^{T}(t \mid T)\right]$ is formulated as follows.

$$
\begin{aligned}
& E\left[e(t \mid T) e^{T}(t \mid T)\right]=\iint_{t}^{T} J(t, s) E\left[v(s) v^{T}(s)\right] J^{T}(t, \tau) \mathrm{d} s \mathrm{~d} \tau \\
& =\int_{t}^{T} J(t, s) R J^{T}(t, s) \mathrm{d} s
\end{aligned}
$$

Let $\mathrm{P}(\mathrm{t} \mid \mathrm{t})$ be the filtering error variance function, which is given by 


$$
\mathrm{P}(\mathrm{t} \mid \mathrm{t})=\mathrm{K}(\mathrm{t}, \mathrm{t})-\mathrm{E}\left[\hat{z}(\mathrm{t} \mid \mathrm{t}) \hat{z}^{T}(\mathrm{t} \mid \mathrm{t})\right]
$$

Putting

$$
\mathrm{U}(\mathrm{t}, \mathrm{T})=\int_{t}^{T} J(t, s) R J^{T}(t, s) \mathrm{d} s,
$$

the fixed-interval smoothing error variance function is given by

$$
\mathrm{P}(\mathrm{t} \mid \mathrm{T})=\mathrm{P}(\mathrm{t} \mid \mathrm{t})-\left(B(t)-A(t) \mathrm{q}_{0}(\mathrm{t})\right) U(t, T)\left(B(t)-A(t) \mathrm{q}_{0}(\mathrm{t})\right)^{T} .
$$

The auto-variance function of the filtering estimate $\hat{z}(\mathrm{t} \mid \mathrm{t}), \mathrm{E}\left[\hat{z}(\mathrm{t} \mid \mathrm{t}) \hat{z}^{T}(\mathrm{t} \mid \mathrm{t})\right]$, is, from (23) and (24),

$$
\begin{aligned}
& \mathrm{E}\left[\hat{z}(\mathrm{t} \mid \mathrm{t}) \hat{z}^{T}(\mathrm{t} \mid \mathrm{t})\right]=A(t) E\left[e_{0}(t) e_{0}^{T}(t)\right] A^{T}(t) \\
& =\mathrm{A}(\mathrm{t}) \int_{0}^{t} J_{0}(s) R J_{0}^{T}(s) \mathrm{d} s A^{T}(t) .
\end{aligned}
$$

From (20), we get

$$
\mathrm{E}\left[\hat{z}(\mathrm{t} \mid \mathrm{t}) \hat{z}^{T}(\mathrm{t} \mid \mathrm{t})\right]=A(t) q_{0}(t) A^{T}(t) .
$$

Differential equation for $\mathrm{q}_{0}(\mathrm{t})$ is given by (14).

Differentiating $\mathrm{U}(\mathrm{t}, \mathrm{T})$ with respect to $\mathrm{t}$ and using (32), we obtain

$$
\frac{d U(t, T)}{d t}=-J(t, t) R J^{T}(t, t)-J(t, t) R J_{0}^{T}(t) U(t, T)-U(t, T) J_{0}(t) J^{T}(t, t), \mathrm{U}(\mathrm{T}, \mathrm{T})=0 .
$$

In summary, the algorithm for the fixed-interval smoothing error variance function $\mathrm{P}(\mathrm{t} \mid \mathrm{T})$ consists of (11), (14), (15) with (34) - (37).

Since $\left(B(t)-A(t) \mathrm{q}_{0}(\mathrm{t})\right) U(t, T)\left(B(t)-A(t) \mathrm{q}_{0}(\mathrm{t})\right)^{T}$ and the filtering error variance function $\mathrm{P}(\mathrm{t} \mid \mathrm{t})$ are positive semidefinite matrices, the following relationship holds.

$$
0 \leq \mathrm{P}(\mathrm{t} \mid \mathrm{T}) \leq \mathrm{P}(\mathrm{t} \mid \mathrm{t}) \leq \mathrm{K}(\mathrm{t}, \mathrm{t})
$$

(38) indicates that the filtering error variance function $\mathrm{P}(\mathrm{t} \mid \mathrm{t})$ is lower bounded by the fixed-interval smoothing error variance function $\mathrm{P}(\mathrm{t} \mid \mathrm{T})$ and upper bounded by the auto-variance function $\mathrm{K}(\mathrm{t}, \mathrm{t})$ of the signal $z(t)$. This fact indicates that the estimation accuracy of the proposed fixed-interval smoother is preferable or equal to that of the filter in Theorem 1 and the proposed fixed-interval smoother and filter are stable for the bounded auto-variance function $\mathrm{K}(\mathrm{t}, \mathrm{t})$.

\section{A Numerical Simulation Example}

Let a scalar observation equation be given by

$$
y(t)=z(t)+v(t) .
$$

Let the observation noise $v(t)$ be a zero-mean white Gaussian process with the variance $R, N(0, R)$. Let the auto-covariance function of the signal $z(t)$ be given by

$$
K(t, s)=\frac{3}{16} e^{-|t-s|}+\frac{5}{48} e^{-3|t-s|} .
$$

From (40), the functions $A(t)$ and $B(s)$ in (3) are expressed as follows:

$$
A(t)=\left[\frac{3}{16} e^{-t} \frac{5}{48} e^{-3 t}\right], B(s)=\left[e^{s} e^{3 s}\right] .
$$

If we substitute (41) into the fixed-interval smoothing algorithm of Theorem 1, we can calculate the fixed-interval smoothing estimate recursively. Fig. 1 illustrates the signal $\mathrm{z}(\mathrm{t})$ and the filtering estimate 
$\hat{z}(t \mid t)$ vs. $\mathrm{t}, 0 \leq \mathrm{t} \leq 2$, for the white Gaussian observation noise $\mathrm{N}\left(0,0.1^{2}\right)$. The filtering estimate, starting with the initial value $\hat{z}(0 \mid 0)=0$ at time $\mathrm{t}=0$, approaches the signal gradually as time $\mathrm{t}$ advances.

Fig. 2 illustrates the signal $z(t)$ and estimates by the proposed fixed-interval smoother in Theorem 1 and the fixed-interval smoother in $[10]$ vs. $t$ for the observation noise $\mathrm{N}\left(0,0.1^{2}\right)$. The fixed-interval smoother, starting from the fixed interval $\mathrm{T}=2$, calculates the fixed-interval smoothing estimate backward to the time $\mathrm{t}=0$. In comparison with the filtering estimate in Fig. 1, the fixed-interval smoother estimates the signal better, in Fig.2, than the filter. Also, from Fig. 2, it is seen that the estimation accuracy of the proposed fixed-interval smoother is preferable to the fixed-interval smoother in [10]. Table 1 shows the mean-square values (MSVs) of estimation errors by the proposed filter, the fiter in [10], the proposed fixed-interval smoother and the fixed-interval smoother in [10] for the observation noises $\mathrm{N}\left(0,0.1^{2}\right), \mathrm{N}\left(0,0.3^{2}\right)$ and $\mathrm{N}\left(0,0.5^{2}\right)$. Here, the MSV of filtering errors is calculated by $\frac{1}{2000} \sum_{i=1}^{2000}(z(i \cdot \Delta)-\hat{z}(i \cdot \Delta \mid i \cdot \Delta))^{2}, \Delta=0.001$. Also, the MSV of fixed-interval smoothing errors is calculated by $\frac{1}{2000} \sum_{i=1}^{2000}(z(i \cdot \Delta)-\hat{z}(i \cdot \Delta \mid 2000 \cdot \Delta))^{2}$. Table 1 indicates, for both the filtering and fixed-interval smoothing estimates, as the variance of the observation noise becomes small, the estimation accuracies are improved. From the MSVs of the estimation errors, it should be noted that the estimation accuracy of the current fixed-interval smoother is preferable to that of the filter. Also, from Table 1, it is seen that the estimation accuracy of the proposed fixed-interval smoother is superior to that in [10]. The MSVs of the filtering errors by the proposed filter and the filter in [10] are same. This is based on the fact that the proposed filtering algorithm in Theorem 1 is same as that in $[10]$.

In the calculations of the filtering and fixed-interval smoothing estimates, as the numerical integration, the fourth-order Runge-Kutta method with the integration step size $\Delta=0.001$ is adopted.

For references, the state-space model, which generates the signal process, is specified by

$$
\begin{aligned}
& z(t)=x_{1}(t), \\
& \frac{d x_{1}(t)}{d t}=x_{2}(t), \frac{d x_{2}(t)}{d t}=-3 x_{1}(t)-4 x_{2}(t)-2 w(t), \\
& E[w(t) w(s)]=\delta(t-s) .
\end{aligned}
$$

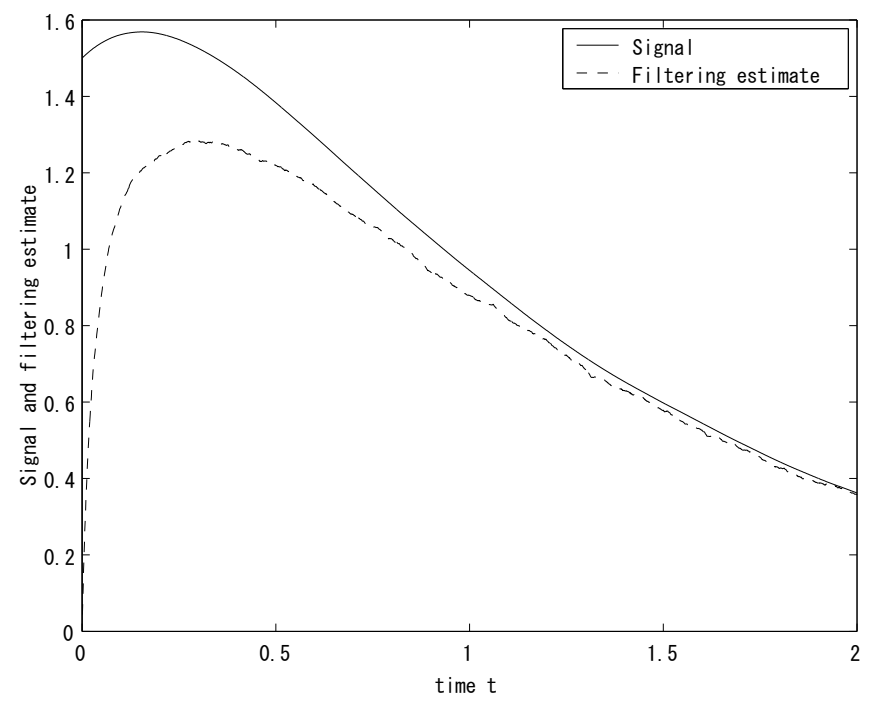

Figure 1. Signal and filtering estimate vs. $t$ for the observation noise $\mathrm{N}\left(0,0.1^{2}\right)$. 


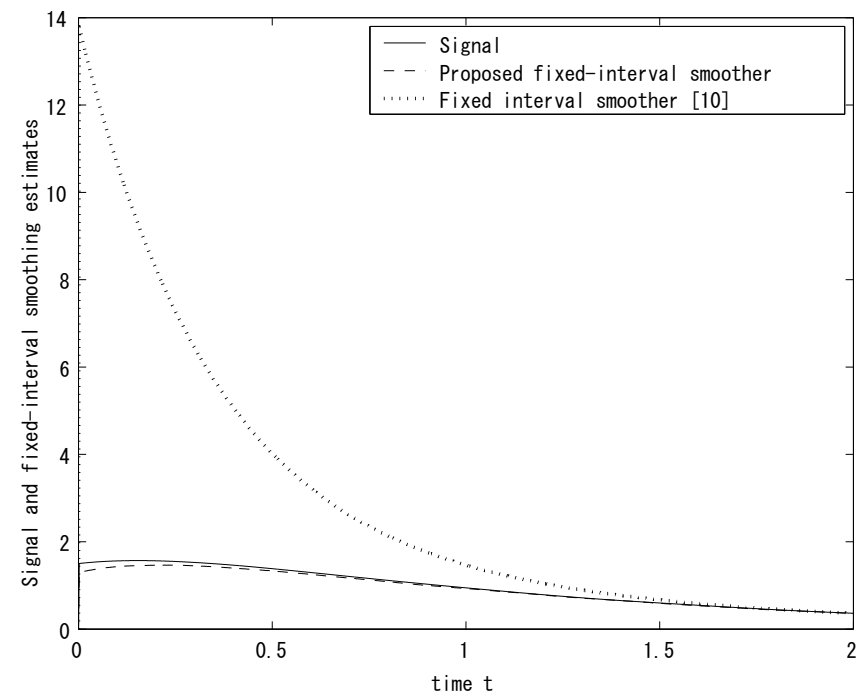

Figure 2. Signal and estimates by the proposed fixed-interval smoother and the fixed-interval smoother in [10] vs. $\mathrm{t}$ for the observation noise $\mathrm{N}\left(0,0.1^{2}\right)$.

Table 1. Mean-square values of estimation errors by the proposed filter, the filter in [10], the proposed fixedinterval smoother and the fixed-interval smoother in [10] for the observation noises $\mathrm{N}\left(0,0.1^{2}\right), \mathrm{N}\left(0,0.3^{2}\right)$ and $\mathrm{N}\left(0,0.5^{2}\right)$.

\begin{tabular}{|l|l|l|l|}
\hline Observation noise & $\begin{array}{l}\text { MSV of filtering errors } \\
\text { by the proposed filter } \\
\text { and the filter in [10] }\end{array}$ & $\begin{array}{l}\text { MSV of estimation errors } \\
\text { by the proposed } \\
\text { fixed-interval smoother }\end{array}$ & $\begin{array}{l}\text { MSV of estimation errors } \\
\text { by the fixed-interval } \\
\text { smoother in [10] }\end{array}$ \\
\hline $\mathrm{N}\left(0,0.1^{2}\right)$ & 0.049031562793425 & 0.003178634946815 & 12.29473997433196 \\
\hline $\mathrm{N}\left(0,0.3^{2}\right)$ & 0.314715422440784 & 0.098148137509625 & 12.97472203403929 \\
\hline $\mathrm{N}\left(0,0.5^{2}\right)$ & 0.573266192619002 & 0.303859037759961 & 8.76674972536291 \\
\hline
\end{tabular}

\section{Conclusions}

This paper, based on the innovation theory, proposed the new fixed-interval smoother using the covariance information in linear continuous-time stochastic systems. From the numerical simulation example in section 5, the fixed-interval smoother, proposed in this paper, improves the estimation accuracy of the filter and its estimation accuracy is superior to that of the fixed-interval smoother in [10]. This indicates that the current fixed-interval smoothing algorithm in Theorem 1 shows the feasible smoothing characteristic.

Also, from the variance of the fixed-interval smoothing errors and the variance of the filtering errors in section 4 , it is shown that the estimation accuracy of the proposed fixed-interval smoother is preferable or equal to that of the filter in Theorem 1.

\section{References}

1. T. Kailath, Lectures on Wiener and Kalman Filtering. Springer-Verlag, 1981.

2. D. Simon, Optimal State Estimation Kalman, $H_{\infty}$, and Nonlinear Approaches. Wiley-Interscience, 2006.

3. S. Nakamori, "Design of recursive Wiener smoother given covariance information," IEICE Trans. Fundamentals of Electronics, Communication and Computer Sciences, vol. E79-A, no. 6, pp. 864-872, 1996.

4. S. Nakamori, A. H. Carazo, and J. L. Pérez, "Design of RLS fixed-lag smoother using covariance information in linear discrete stochastic systems," Applied Mathematical Modelling, vol. 34, no. 4, pp. 1093-1106, 2010.

5. S. Nakamori, A. H. Carazo, and J. L. Pérez, "Design of fixed-lag smoother using covariance information based on innovations approach in linear discrete-time stochastic systems," Applied Mathematics and Computation, vol. 193, no. 1, pp. 162-174, 2007. 
6. S. Nakamori, A. H. Carazo, and J. L. Pérez, "Design of RLS Wiener fixed-lag smoother using covariance information in linear discrete stochastic systems," Applied Mathematical Modelling, vol. 32, no. 7, pp. 1338-1349, 2008.

7. S. Nakamori, "Recursive least squares fixed-lag Wiener smoothing using autoregressive signal models for linear discrete-time systems," Applied Mathematical Modelling, vol. 39, no. 21, pp. 6451-6460, 2015.

8. T. Kailath and P. Frost, "An innovation approach to least-squares estimation part II: Linear smoothing in additive white noise," IEEE Trans.Automatic Control, vol. AC-13, no. 6, pp. 655-660, 1968.

9. S. Nakamori, "RLS fixed-lag smoother using covariance information based on innovation approach in linear continuous stochastic systems," Journal of Information, vol. 1, no. 2, pp. 23-35, 2015.

10. S. Nakamori, "New design of fixed-interval smoother using covariance information in linear stochastic continuous-time systems," Applied Mathematics and Computation, vol. 144, no. 2, pp. 557-567, 2003.

11. S. Fujita and T. Fukao, "Optimal linear fixed-interval smoothing for colored noise," Information and Control, vol. 17, pp. 313-325, 1970.

12. S. Nakamori, A. H. Carazo, and J. L. Pérez, "Design of a fixed-interval smoother using covariance information based on the innovations approach in linear discrete-time stochastic systems," Applied Mathematical Modelling, vol. 30 , pp. 406-417, 2006.

13. S. Nakamori, "RLS Wiener smoother for colored observation noise with relation to innovation theory in linear discrete-time stochastic systems," I.J. Information Technology and Computer Science, vol. 5, no. 3, pp. 1-12, 2013.

14. A. P. Sage and J. L. Melsa, Estimation Theory with Applications to Communications and Control. McGrawHill, 1971. 